\title{
Exploring the Treatment of Coronary Artery Atherosclerosis from the Direction of Inflammatory Vesicles with Wind-Extinguishing and Phlegm-Resolving Medicine
}

\author{
Bing Zhao, Lihong Gong* \\ Liaoning University of Traditional Chinese Medicine, Shenyang 110032, China \\ *Corresponding author: Lihong Gong, linda1795@sina.com
}

Copyright: (C) 2022 Author(s). This is an open-access article distributed under the terms of the Creative Commons Attribution License (CC BY 4.0), permitting distribution and reproduction in any medium, provided the original work is cited.

\begin{abstract}
Atherosclerosis is a fundamental pathological change in coronary heart disease, with vulnerable plaque formation leading to decreased plaque stability and plaque rupture, and is a major cause of acute cardiovascular events. The inflammatory response is an important mechanism in the development and progression of atherosclerosis, and plaque stability is closely related to the inflammatory response. In recent years, the role of NLRP3 inflammatory vesicles, which are involved in macrophage foaminess, in the inflammatory response to atherosclerosis has received increasing attention. Chinese medicine is able to modulate inflammatory vesicles to improve atherosclerotic plaque stability, showing good promise in the prevention and treatment of atherosclerotic vulnerable plaques. Based on the role of inflammatory vesicles in atherosclerosis and the results of previous studies that wind-extinguishing and phlegm-resolving medicine can effectively modulate the inflammatory response to intervene in the treatment of atherosclerosis, this paper aims to investigate the treatment of coronary atherosclerosis by wind-extinguishing and phlegm-resolving medicine from the direction of inflammatory vesicles.
\end{abstract}

Keywords: Atherosclerosis; Macrophages; Inflammatory vesicles; Wind-Extinguishing and Phlegm-Resolving Medicine

Online publication: January 19, 2022

\section{Introduction}

Atherosclerosis (AS) is the main pathological basis of atherosclerotic cardiovascular diseases such as coronary heart disease and stroke, and its prevalence is increasing and becoming younger as living standards improve. Stabilization of plaque is of great importance as the rupture of unstable plaque, secondary to thrombosis and lumen occlusion, is the main pathological basis of acute coronary syndromes. The inflammatory response plays a pivotal role in the pathological process of AS. Inflammasomes are multiprotein complexes, of which NLRP3 inflammasomes are the most representative ones, involved in the atherosclerotic inflammatory response. Previous research by Prof. Gong Lihong's team has demonstrated the anti-atherosclerotic effect of wind-extinguishing medicinal compounds, the mechanism of which is related to the inhibition of the body's inflammatory response ${ }^{[1]}$. This paper is based on the results of a previous study conducted by the group on the ${ }^{[2,3]}$ effective intervention of wind-extinguishing and phlegmresolving medicinal in AS, and is intended to further explore the feasibility of wind-extinguishing and phlegm-resolving medicine in the prevention and treatment of inflammatory vesicles in AS. 


\section{Advances in the study of the inflammatory response to $\mathrm{AS}$}

AS is a chronic inflammatory disease that occurs in the arterial blood vessel wall ${ }^{[4]}$. The pathogenesis of AS is not well understood, but the inflammatory response is a central factor in the pathogenesis of AS and plays an important role in its development. It is generally accepted that the inflammatory response in AS is regulated by both innate and adaptive immunity, and that immune cells such as macrophages are involved in the inflammatory response and disease development in AS, with targets for intervention including CRP, TNF-a and IL-6. The CANTOS study was the first to validate the feasibility ${ }^{[5]}$ of IL- $1 \beta$ as a target for the treatment of coronary artery disease, marking the formal elevation of the "inflammatory hypothesis" of AS to an "inflammatory theory." "The study also showed that the inhibition of IL-1 $\beta$ as a target for coronary artery disease was indiscriminate. At the same time, studies have shown that indiscriminate inhibition of IL-1 $\beta$ may increase the chance of infection and that biologic agents targeting IL-1 $\beta$ are expensive, so targeting IL- $1 \beta$ upstream is urgent. The role of NLRP 3 inflammatory vesicles in the inflammatory response to AS has received increasing attention in recent years, as they regulate downstream IL-1 $\beta$ activity and are involved in macrophage foaminess ${ }^{[6]}$. Therefore, anti-inflammatory therapy targeting NLRP3 inflammatory vesicles is currently an important therapeutic measure to alleviate AS.

\section{NLRP3 inflammatory vesicles in AS macrophages}

Inflammatory vesicles are multiprotein complexes formed by the assembly of intracytoplasmic pattern recognition receptors involved in the recognition of pathogen-associated molecular patterns that activate cysteine aspartate-proteinase-1 (Caspase-1), which in turn regulates IL-1 $\beta$ and IL-18 levels ${ }^{[7]}$. Nucleotidebinding oligomerization domain-like receptor 3 (NLRP3) is a major inflammatory vesicle in cardiovascular disease research. NLRP3 inflammatory vesicles are a complex of NLRP3, apoptosis-associated specklelike protein (ASC) and pro-Caspase-1. Inhibition of NLRP3 inflammasomes has been demonstrated to help limit the inflammatory response ${ }^{[8]}$, and NLRP3 inflammasome levels are positively linked with IL-1 and IL-18 levels. The pro-inflammatory development of cardiovascular disease is hypothesized to be linked to NF-kB signaling. NF-kB is initially activated to enhance pro-IL-1 and NLRP3 expression ${ }^{[9]}$. Signals from $\mathrm{NF}-\mathrm{kB}$ activators and a second trigger, such as ROS generation by mitochondria, are required for the activation of NLRP3 inflammatory vesicles ${ }^{[10]}$. In vitro studies have shown that NLRP3 inflammatory vesicles can be activated and secrete IL- 1 in THP- 1 cells ${ }^{[11]}$, and in vivo studies have shown that NLRP3 inflammatory vesicles can be activated and secrete IL-1 in THP-1 cells. NLRP3-containing inflammatory vesicles causes ${ }^{[12]}$ Plaque development to be stopped, pro-inflammatory cytokine expression to be inhibited, plaque lipid content to be reduced, and plaque to be stabilized when the NLRP3 gene is silenced ${ }^{[13]}$. Blocking IL-1R on endothelial cells reduces particle activity and inhibits particle-dependent activation of cell adhesion molecules in THP-1 monocytes ${ }^{[14]}$. In peripheral blood mononuclear cells from patients with coronary artery disease, atorvastatin inhibited the expression of NLRP3 inflammatory vesicles, suggesting that inhibition of NLRP3 inflammatory vesicles may contribute to the inhibitory effect of atorvastatin on chronic inflammation and AS progression. According to the findings, NLRP3 inflammatory vesicles are increased during AS, and NLRP3 expression levels are positively related with AS risk factors and significant adverse cardiac events. The above results suggest that NLRP3 inflammatory vesicles are upregulated during AS and that NLRP3 expression levels are positively associated with AS risk factors and serious adverse cardiac events.

\section{Correlation between "wind-seeking and expectorant herbs" and inflammatory vesicles}

AS can be classified as "chest paralysis" or "true heart pain" according to its clinical manifestations. Treatment is usually based on activating blood stasis, warming the meridians and dispersing cold, clearing phlegm and etc. ${ }^{[15]}$. Based on the concept of "cardiovascular event chain" in Western medicine, Professor 
Gong Lihong proposed the hypothesis of AS event chain in Chinese medicine: "deficiency - phlegm - stasis - toxicity (heat) - wind." The wind-phlegm theory is an innovative explanation for the occurrence and development of AS ${ }^{[16]}$. The patient's reduced exercise and diet of thick and fatty foods led to spleen deficiency and phlegm, phlegm clotting and blood stasis, phlegm and stagnation of phlegm and stagnation of blood that did not resolve and led to internal carbuncle and toxic heat, and heat that led to internal wind, and wind, which is the longest of all diseases, is good at moving and changing several times, and phlegm and wind move internally ${ }^{[17,18]}$. By establishing an ApoE knockout mouse coronary artery model, Chi Yingxue found that the Chinese herbal remedy, Complex Stable Plaque Soup, might reduce inflammation by affecting the expression of HO-1 and PPAR $\gamma$, thereby interfering with AS plaque formation and rupture [19].

Professor Gong Lihong established an angiotensin II-induced human umbilical vein endothelial cell model and a high-fat diet-induced ApoE knockout mouse model, and found that wind-extinguishing and phlegm-resolving medicine could inhibit Ang II-induced endothelial cell injury, and effectively reduce NF$\mathrm{kB}$ mRNA expression and TLR4 mRNA expression in the abdominal aorta of AS mice, thereby inhibiting the inflammatory response and stabilizing plaque ${ }^{[20,21]}$. Ni Xiao-Ou and Gong Li-Hong showed that the ApoE knockout mouse coronary artery model could increase the protein expression of beclin-1 and LC3, improve cellular autophagy, and affect the inflammatory response. The Chinese herbal remedy Sowing Wind and Expectorant Spots can inhibit the inflammatory ${ }^{[22]}$ response within the plaques and upregulate the expression of PPAR- $\gamma$ gene in ApoE knockout mice with experimental AS unstable plaques ${ }^{[23]}$. Therefore, it is quite feasible to use wind-extinguishing and phlegm-resolving medicinal to combat inflammatory vesicles in AS based on the theory of wind and phlegm.

Inhibition of inflammatory vesicles through various pathways in the early stages of AS can intervene in the development process of $\mathrm{AS}^{[24]}$, reducing plaque production. On this basis, further animal experiments can be carried out to confirm the association between Searching for Wind and Expectorant Herbs and inflammatory vesicles, which is a step we need to refine. Professor Gong Lihong believes that "windphlegm" is the main cause of AS, similar to unstable plaques in Western medicine ${ }^{[25,26]}$. Chen Pi, Radix et Rhizoma Atractylodis Macrocephala and Rhizoma Atractylodis Macrocephala are used together to dissolve phlegm and disperse knots, as well as to dry dampness and remove lumps, and are the secondary herbs; Leech helps the primary herbs to break up blood and open the channels, and is the secondary herb. In modern pharmacological studies, the whole scorpion contains the active peptide "SVC-IV" with analgesic and anti-inflammatory effects ${ }^{[27]}$. The centipede contains analgesic peptide monomers with analgesic and anti-inflammatory effects ${ }^{[28]}$. Zhang Fengchun et al. ${ }^{[29]}$ conducted experimental studies using mice and found that the extract of Dilong's liquid had a significant promotion effect on the activity of macrophage FC receptors, accelerating the speed of phagocytosis of neutrophils and thus playing an anti-inflammatory role. Chen Pi contains cellulose, which has anti-tumor, anti-inflammatory, neuroprotective, hepatorenal and obesity inhibiting effects, and has certain intervention effects in diabetes, asthma and cardiovascular diseases ${ }^{[30]}$. The half of Atractylodis Macrocephalae contains alkaloids, organic acids, polysaccharides, sterols and other chemical components, which have pharmacological effects such as anti-cough and asthma, anti-inflammatory, anti-tumor and anti-vomiting ${ }^{[31]}$. Atractylenolide, as well as Atractylodes polysaccharides, have anti-inflammatory and modulating immune metabolism effects ${ }^{[32]}$. Leeches are effective in reducing the level of inflammatory factor TNF- $\alpha$ and inhibiting the proliferation of vascular smooth muscle cells (SMC), thereby reducing the size of atherosclerotic (AS) plaques ${ }^{[33]}$.

5. Feasibility of using wind and phlegm-seeking herbs to prevent and treat inflammatory vesicles in AS based on the theory of wind and phlegm

There is support in the literature for a study on the mechanism of NLRP3 inflammatory vesicles in AS with 
the formula that is wind-extinguishing and phlegm-resolving: Chen Ning et al ${ }^{[34]}$ established a model of AS by high-fat diet combined with immune damage method, and after 8 weeks were given the formula to resolve phlegm and dispel stasis. The results showed that the formula to resolve phlegm and dispel stasis could reduce fat vacuoles, restore liver tissue morphology, reduce NLRP3 protein content and serum IL-1 $\beta$ and IL-18 content, thus improving the results showed that the treatment of phlegm and elimination of stasis could reduce fat vacuoles, restore liver tissue morphology, reduce NLRP3 protein content and serum IL-1 $\beta$ and IL-18 levels, thus improving liver lipid deposition in AS rabbits ${ }^{[35]}$. The study found that compound Astragalus capsule could reduce NLRP3 content, inhibit inflammatory response, improve carotid endothelial damage and reduce carotid atherosclerosis. Jiang ${ }^{[36]}$ used a high-fat diet combined with intraperitoneal injection of vitamin $\mathrm{D}_{3}$ to establish a rat AS model, and administered the drug after molding. The aqueous extract of Centipede exerted anti-AS effects by ${ }^{[37]}$ inhibiting the value-added and division of smooth muscle cells. Hemiptera exerted anti-AS effects by regulating the PI3K/Akt signaling pathway ${ }^{\text {[38] }}$. Chuan Chen Pi reduced LPS-stimulated RAW264.7 cell injury through the TLR4-NF-kB signaling pathway ${ }^{[39]}$ and leech extract inhibited NF-kB expression to combat AS ${ }^{[40]}$.

In summary, based on a series of systematic studies on the prevention and treatment of AS with windextinguishing and phlegm-resolving medicinal, it has been clearly proposed that wind-extinguishing and phlegm-resolving medicinal may effectively prevent and treat AS by regulating the inflammatory response, but the effect of wind-extinguishing and phlegm-resolving medicine on NLRP3 inflammatory vesicles in AS has not been elucidated. The inflammatory response and wind-phlegm theory are correlated, and NLRP3 inflammatory vesicles are valued in the inflammatory response of AS, and the process of AS development can be inhibited by reducing NLRP3 inflammatory vesicles. Based on this, wind and phlegm dispelling herbs may have an effective role in regulating NLRP3 inflammatory vesicles, thus opening up new perspectives on the regulatory mechanism for their effective intervention in the treatment of AS. Combined with the key targets of NLRP3 inflammatory vesicles and related signaling pathways in the formation of $\mathrm{AS}$, and in close connection with the results of previous studies on the effective intervention of wind-extinguishing and phlegm-resolving medicinal in AS, we can provide a multi-level and multi-target scientific basis for the effective intervention of wind-extinguishing and phlegm-resolving medicine in AS, which is expected to provide an experimental basis for the "wind-extinguishing and phlegm-resolving medicine" treatment of AS. It is expected to provide an experimental basis for the treatment of AS by the "Searching Wind and Dispelling Phlegm Method" and help enrich the scientific connotation of the theory of "wind and phlegm."

\section{Disclosure statement}

The author declares no conflict of interest.

\section{References}

[1] Zhao L, Gong L, 2017, The Clinical Efficacy of Compound Wenban Decoction Combined with External Counterpulsation on Patients with Unstable Angina Pectoris of Coronary Heart Disease and the Effect on Serum Nitric Oxide and Vascular Endothelin-1. Integrated Chinese and Western Medicine Journal of Cerebrovascular Diseases, 15(18): 2228-2231.

[2] Zhao D, Gong L, Xiao F, 2018, The Effect of Wenban Decoction Containing Serum on the Expression of Macrophage Foaming Autophagy-Related Proteins LC3B and Beclin 1. Journal of Traditional Chinese Medicine, 59(11): 978-982.

[3] Zhang S, Gong L, Ni X, et al., 2015, Effect of Wind-Extinguishing and Phlegm-Resolving Medicinal Compound on the Expression of Beclin-1 and Bcl-2 Genes in Atherosclerotic Vulnerable Plaques of AopE Gene Knockout Mice. Chinese Journal of Traditional Chinese Medicine, 33(9): 2151-2153. 
[4] Wolf D, Ley K, 2019, Immunity and Inflammation in Atherosclerosis.Circ Res, 124(2): 315-327.

[5] Ridker PM, Everett BM, Thuren T, et al., 2017, Antiinflammation Therapy with Canakinumab for Atherosclerotic Disease. N EngI J Med, 377(12): 1119-1131.

[6] Liu W, Yin Y, Zhou Z, et al., 2014, OxLDL-Induced IL-1 Beta Secretion Promoting foam Cells Formation was Mainly via CD36 Mediated ROS Production Leading to NLRP3 Inflammasome Activation. Inflamm Res, 63(1):33-43.

[7] Lamkanfi M, Dixit VM, 2014, Mechanisms and Functions of Inflammasomes. Cell, 157(5): 1013-1022.

[8] Yan Y, Jiang W, Liu L, et al., 2015, Dopamine Controls Systemic Inflammation Through Inhibition of NLRP3 Inflammasome. Cell, 160: 62-73.

[9] Volt H, García JA, Doerrier C, et al., 2016, Same Molecule but Different Expression: Aging and Sepsis Trigger NLRP3 Inflammasome Activation, a Target of Melatonin. J Pineal Res, 60(2): 193-205.

[10] Zhou R, Yazdi AS, Menu P, et al., 2011, A Role for Mitochondria in NLRP3 Inflammasome Activation. Nature, 469: 221-225.

[11] He D, Wang H, Xu L, et al., 2016, Saikosaponin-A Attenuates Oxidized LDL Uptake and Prompts Cholesterol Efflux in THP-1 Cells. J Cardiovasc Pharmacol, 67(6): 510 -518.

[12] Satoh M, Tabuchi T, Itoh T, et al., 2014, NLRP3 Inflammasome Activation in Coronary Artery Disease: Results from Prospective and Randomized Study of Treatment with Atorvastatin or Rosuvastatin. Clinical Science, 126(3-4): 233-241.

[13] Zheng F, Xing S, Gong Z, et al., 2014, Silence of NLRP3 Suppresses Atherosclerosis and Stabilizes Plaques in Apolipoprotein E-deficient Mice. Mediators of Inflammation, 2014: 1-8.

[14] Ehsan M, Singh KK, Lovren F, et al., 2016, Adiponectin Limits Monocytic Microparticle-Induced Endothelial Activation by Modulation of the AMPK, Akt and NFאB Signaling Pathways. Atherosclerosis, 245: 1-11.

[15] Cardiovascular Branch of Chinese Society of Traditional Chinese Medicine, 2018, Expert Consensus on Diagnosis and Treatment of Stable Angina Pectoris in Coronary Heart Disease. Journal of Traditional Chinese Medicine, 59(5): 447-450.

[16] Yan X, Gong L, 2019, Treating Coronary Heart Disease from the Perspective of the "Deficiency-PhlegmStasis-Toxin (Heat)-Wind" Event Chain. Journal of Integrated Traditional Chinese and Western Medicine Cardio-Cerebrovascular Disease, 17(13): 1985-1987.

[17] Guo Q, 2012, Professor Gong Lihong's Experience in Treating Unstable Angina Pectoris based on Wind Phlegm Theory. Shenyang: Liaoning University of Traditional Chinese Medicine.

[18] Nourooz-Zadeh J, Smith CC, Betteridge DJ, 2001, Measures of Oxidative Stress in Heterozygous Familial Hypercholesterolaemia. Atherosclerosis, 156(2): 435-441.

[19] Chi Y, Gong L, 2015, Effect of Wind-Extinguishing and Phlegm-Resolving Medicinal Compound on the Expression of HO-1 and PPAR $\gamma$ in Atherosclerotic Unstable Plaques of Apo E Gene Knockout Mice. Chinese Emergency in Traditional Chinese Medicine, 24(1): 4-6.

[20] Jiang M, Gong L, 2015, Effect of Wenban Decoction on TLR4 mRNA in Atherosclerotic Unstable Plaques of ApoE Knockout Mice. Journal of Traditional Chinese Medicine, 56(7): 598-601.

[21] Ding Y, Gong L, Gao F, et al., 2010, The Effect of Chinese Herbal Medicine for Seeking Wind and Expelling Phlegm on the Expression of NF-kB mRNA and ET-1 mRNA in Human Umbilical Vein Endothelial Cells Induced by Ang II. Integrated Traditional Chinese and Western Medicine Disease Journal, 8(12): 1479-1481.

[22] Ni X, Gong L, Zhang S, et al., 2016, The Effect of Wind-Extinguishing and Phlegm-Resolving Medicinal on the Autophagy-Related Proteins Beclin1 and LC3 of Atherosclerotic Unstable Plaque in ApoE Gene Knockout Mice. Chinese and Western Medicine Combined Cardio-Cerebrovascular Disease, 14(05): 488491.

[23] An Y, Gong L, Wei W, 2014, The Effect of Wind-Searching and Expectorating Method on Inflammatory Response and PPAR $\gamma$ Gene Expression in Aortic Vulnerable Plaques of ApoE Gene Knockout Mice. 
Chinese Emergency in Traditional Chinese Medicine, 23(09): 1591-1593+1609.

[24] Han J, Pan XY, Xu Y, et al., 2012, Curcumin Induces Autophagy to Protect Vascular Endothelial Cell Survival from Oxidative Stress Damage. Autophagy, 8(5): 812-825.

[25] Guo Q, 2012, Professor Gong Lihong's Experience in Treating Unstable Angina Pectoris based on Wind Phlegm Theory. Shenyang: Liaoning University of Traditional Chinese Medicine.

[26] Cheng X, Gong L, Li N, et al., 2016, Effect of Wenban Decoction on the Methylation of Plasma Thrombomodulin Gene Promoter in Patients with Acute Coronary Syndrome with Phlegm and Blood Stasis Syndrome. Journal of Traditional Chinese Medicine, 57(2): 140-144.

[27] Han X, Xu X, Shen Q, et al., 1996, Research on the Separation and Purification of Scorpion Toxin IV. Journal of Henan Medical University, (03): 1-4.

[28] Zou J, 2010, Extraction and Isolation of Centipede Polypeptide and Study on Its Analgesic Activity. Hubei University of Traditional Chinese Medicine.

[29] Tuesday Fu, 2014, The Modern Pharmacology and Common Clinical Compatibility Analysis of Dilong. China Modern Medicine, 8(03): 238-239.

[30] Xiong Y, Fang Y, Han Y, et al., 2018, Research Progress in the Pharmacological Effects of Tangerine. Chinese Patent Medicine, 40(09): 2030-2033.

[31] Yang L, Zhou Y, Wang X, et al., 2021, Research Progress on the Influence of Processing on the Chemical Constituents and Pharmacological Effects of Pinellia Ternata. Journal of Liaoning University of Traditional Chinese Medicine. 1-13.

[32] Zuo J, Zhang J, Hu X, 2021, Research Progress on the Chemical Constituents and Modern Pharmacological Effects of Atractylodes Macrocephala. Journal of Liaoning University of Traditional Chinese Medicine. 18.

[33] Nie Y, Shen L, He J, et al., 2014, Observation on the Effect of Leech Extract on Inflammation of Rat Epithelial Tissue. China Health Industry, 11(09): 24-26.

[34] Chen N, Jia L, Song N, et al., 2019, Effect of Huatan Quyu Recipe on Lipid Deposition in the Liver of Atherosclerotic Rabbits. Journal of Traditional Chinese Medicine, 60(20): 1755-1759.

[35] Liang W, 2018, Study on the Efficacy and Mechanism of Fufang Qima Capsule in Improving Carotid Artery Intimal Thickening. Guangzhou: Guangzhou University of Traditional Chinese Medicine.

[36] Jiang S, 2019, Anti-Atherosclerosis Effect and Mechanism of Smilax China. Hubei University of Traditional Chinese Medicine, Wuhan.

[37] Zhang Y, Si Q, Wang X, 2005, Experimental Study of Centipede Against Atherosclerosis in Rabbits. Pharmacology and Clinics of Chinese Materia Medica, 21(1): 26-27.

[38] Sun Y, Huang X, Liang W, et al., 2018, Effects of Dried Tangerine Peel and Pinellia on PI3K-Akt Pathway, SOD, MDA, SA- $\beta$-gal Levels in Atherosclerotic Mice. Journal of Capital Medical University, 39(6): $805-$ 809.

[39] Chen J, 2019, Protective Efect of Chuanpisu on LPS-Induced RAW 264.7 Cell Damage. Modern Food Science and Technology, 35(10): 44-49.

[40] Yao W, Xin Z, Wang YS, et al., 2014, An Extract from Medical Leech Improve the Function of Endothelial Cells In Vitro and Attenuates Atherosclerosis in ApoE Null Mice by Reducing Macrophages in the Lesions. Biochemical and Biophysical Research Communications, 455(2): 119-125.

\section{Publisher's note}

Bio-Byword Scientific Publishing remains neutral with regard to jurisdictional claims in published maps and institutional affiliations. 